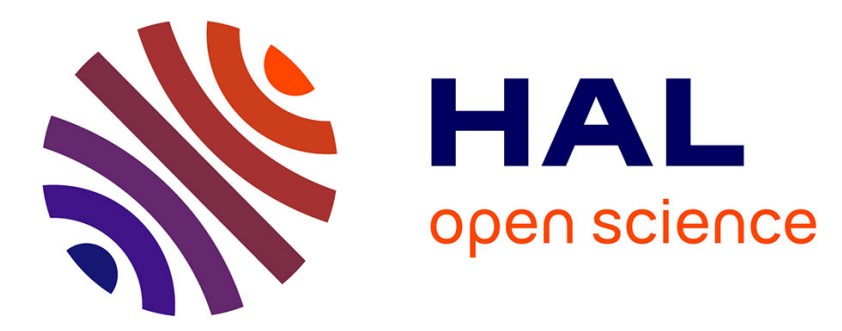

\title{
Déplacement et élargissement de la transition à deux photons 3S-4D dans l'atome de sodium par collision contre des atomes de néon
}

\author{
F. Biraben, B. Cagnac, G. Grynberg
}

\section{- To cite this version:}

F. Biraben, B. Cagnac, G. Grynberg. Déplacement et élargissement de la transition à deux photons 3S-4D dans l'atome de sodium par collision contre des atomes de néon. Journal de Physique Lettres, 1975, 36 (2), pp.41-43. 10.1051/jphyslet:0197500360204100 . jpa-00231149

HAL Id: jpa-00231149

https://hal.science/jpa-00231149

Submitted on 1 Jan 1975

HAL is a multi-disciplinary open access archive for the deposit and dissemination of scientific research documents, whether they are published or not. The documents may come from teaching and research institutions in France or abroad, or from public or private research centers.
L'archive ouverte pluridisciplinaire HAL, est destinée au dépôt et à la diffusion de documents scientifiques de niveau recherche, publiés ou non, émanant des établissements d'enseignement et de recherche français ou étrangers, des laboratoires publics ou privés. 


\title{
DÉPLACEMENT ET ÉLARGISSEMENT DE LA TRANSITION A DEUX PHOTONS 3S-4D DANS L'ATOME DE SODIUM PAR COLLISION CONTRE DES ATOMES DE NÉON
}

\author{
F. BIRABEN, B. CAGNAC et G. GRYNBERG \\ Laboratoire de Spectroscopie Hertzienne de l'ENS (*) \\ Université Pierre-et-Marie-Curie, 75231 Paris Cedex 05, France
}

(Reşu le 5 décembre 1974, accepté le 12 décembre 1974)

\begin{abstract}
Résumé. - On étudie expérimentalement l'élargissement et le déplacement de la transition à deux photons sans élargissement Doppler 3S-4D dans l'atome de sodium par collision contre des atomes de néon. On trouve pour l'élargissement $\alpha=32 \pm 5 \mathrm{MHz}$ /torr et pour le déplacement $\beta=-7 \pm 1 \mathrm{MHz} /$ torr. Cette méthode permet d'étudier l'effet des collisions dans des niveaux $\mathrm{S}$ et $\mathrm{D}$ avec de faibles pressions de gaz étrangers (environ 1 torr).
\end{abstract}

\begin{abstract}
We investigate experimentally the broadening and the shift of the 3S-4D twophoton transition without Doppler broadening in atomic sodium in collision with neon atoms. We find $\alpha=32 \pm 5 \mathrm{MHz}$ /torr for the broadening and $\beta=-7 \pm 1 \mathrm{MHz} /$ torr for the shift. This method can be used for investigating the effect of collisions on $S$ and $D$ levels and it needs low pressures of foreign gases (about 1 torr).
\end{abstract}

Depuis leur mise en évidence $[1,2,3]$, les transitions à deux photons sans élargissement Doppler $[4,5]$ ont été essentiellement utilisées pour mesurer des structures fines et hyperfines $[6,7,8]$. Nous montrons dans cette lettre que ces transitions permettent également d'obtenir des résultats intéressants dans l'étude des collisions à basse pression. Grâce à l'élimination de l'élargissement Doppler, et à l'utilisation d'un LASER à colorant monomode, on peut observer des spectres d'excitation, formés de raies très fines, dont la largeur est réduite à environ $10 \mathrm{MHz}$. Ceci permet de mesurer avec précision des élargissements et des déplacements de niveaux de l'ordre de quelques $\mathrm{MHz}$, produits par des gaz à faibles pressions. Il convient également de remarquer que cette méthode permet d'étendre l'étude des collisions à des niveaux de même parité que le niveau fondamental qui ne peuvent évidemment pas être atteints par absorption d'une raie de résonance [9].

L'expérience a été faite sur la transition 3S-4D du sodium en présence de néon. L'étude du spectre d'excitation en absence de gaz étranger est décrite dans de précédentes publications $[3,10,11]$; ce spectre est décomposé en quatre raies correspondant aux transitions entre les deux sous-niveaux hyperfins $F=2$ et $F=1$ du niveau fondamental et les deux niveaux de structure fine $J^{\prime}=\frac{5}{2}$ et $J^{\prime}=\frac{3}{2}$ de l'état

(*) Associé au CNRS excité. Ces quatres résonances sont désignées comme dans les précédentes publications par :

$$
\begin{aligned}
& 3 \mathrm{~S}, F=2 \rightarrow 4 \mathrm{D}, J^{\prime}=\frac{5}{2} \\
& 3 \mathrm{~S}, F=2 \rightarrow 4 \mathrm{D}, J^{\prime}=\frac{3}{2} \\
& 3 \mathrm{~S}, F=1 \rightarrow 4 \mathrm{D}, J^{\prime}=\frac{5}{2} \\
& 3 \mathrm{~S}, F=1 \rightarrow 4 \mathrm{D}, J^{\prime}=\frac{3}{2} .
\end{aligned}
$$

En présence de néon, ces résonances s'élargissent et se déplacent. Afin de mesurer avec précision ce déplacement nous avons utilisé le montage expérimental présenté sur la figure 1 : le faisceau du laser à colorant est divisé en deux par une lame séparatrice; le faisceau 1, dont l'intensité est $30 \%$ de l'intensité du faisceau incident, traverse une cellule de sodium pur et est renvoyé sur lui-même à l'aide du miroir $\mathbf{M}_{1}$ centré

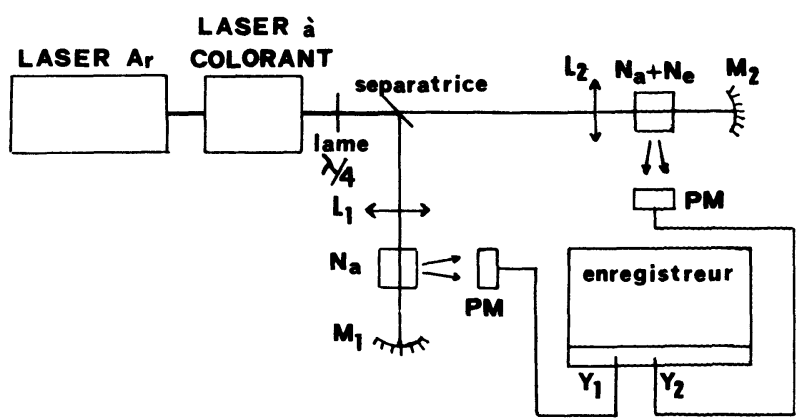

Fig. 1. - Schéma du montage expérimental. 
au foyer de la lentille $L_{1}$; le faisceau 2 traverse une cellule contenant du sodium et du néon et est renvoyé sur lui-même grâce au miroir $\mathbf{M}_{2}$. La lumière de fluorescence provenant des deux cellules est reçue par deux photomultiplicateurs dont les courants sont simultanément mesurés sur les voies $\mathrm{Y}_{1}$ et $\mathrm{Y}_{2}$ du même enregistreur. Comme signal de fluorescence, nous avons observé soit la transition $4 \mathrm{P} \rightarrow 3 \mathrm{~S}$ à $3302 \AA$ provenant d'une cascade du niveau 4D, soit la transition directe $4 \mathrm{D} \rightarrow 3 \mathrm{P}(5682 \AA$ et $5688 \AA)$. Les autres éléments du montage expérimental sont semblables à ceux décrits dans de précédentes publications $[7,11]$.

L'enregistrement simultané des signaux fournis par la cellule 1 remplie de sodium pur et par la cellule 2 contenant en outre un gaz étranger, nous oblige à diviser la puissance du LASER et a pour conséquence de diminuer la qualité des signaux observés. Mais cet enregistrement simultané permet de mettre en évidence de manière certaine de faibles élargissements et surtout de faibles déplacements. Il convient, en outre, de remarquer que la précision des mesures est limitée par les non-linéarités du balayage piézoélectrique du LASER (qui peuvent atteindre $15 \%$ ); ces non-linéarités ne peuvent cependant mettre en cause ni le signe ni l'ordre de grandeur des effets observés.

La figure 2 présente un enregistrement typique obtenu lorsqu'on balaye la fréquence du LASER en fonction du temps. Le signal de fluorescence de la cellule de sodium pur est tracé sur la voie $Y_{1}$, tandis que sur la voie $Y_{2}$ est tracé le signal provenant d'une cellule contenant du sodium et 1 torr de néon (la pression de néon est. mesurée à $20^{\circ} \mathrm{C}$ alors que la température de travail de la cellule 2 est $140^{\circ} \mathrm{C}$ ). Les résonances (a), (b), (c) et (d) sur la voie $Y_{1}$ ont une largeur de $10 \mathrm{MHz}$ (la majeure partie de cette largeur est due au jitter du LASER); les résonances sur la voie $Y_{2}$ sont élargies et légèrement déplacées.
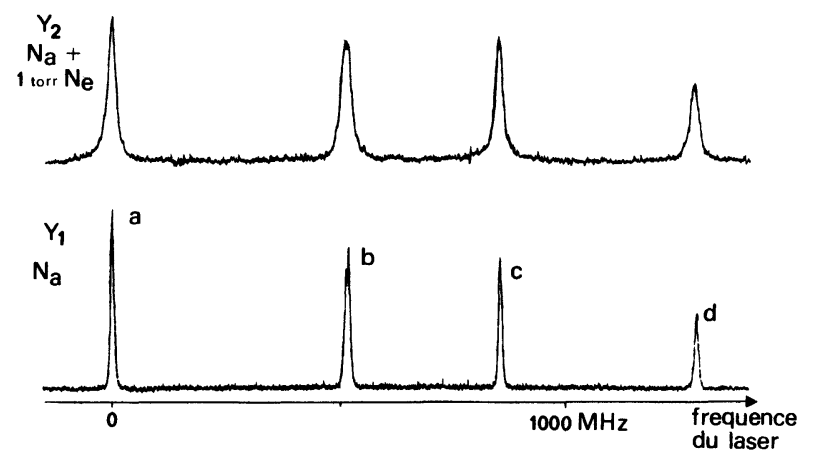

FIG. 2. - Enregistrement simultané du spectre d'excitation à deux photons pour une cellule de $\mathrm{Na}$ sans gaz étranger (voie $\mathrm{Y}_{1}$ ) et pour une cellule contenant $\mathrm{Na}$ et 1 torr de $\mathrm{Ne}$ (voie $\mathrm{Y}_{2}$ ).

Ce déplacement du centre de la résonance s'observe de façon plus nette sur des enregistrements à échelle dilatée, ou bien lorsque la cellule est remplie avec 5 torr de néon. La figure 3 présente avec une échelle

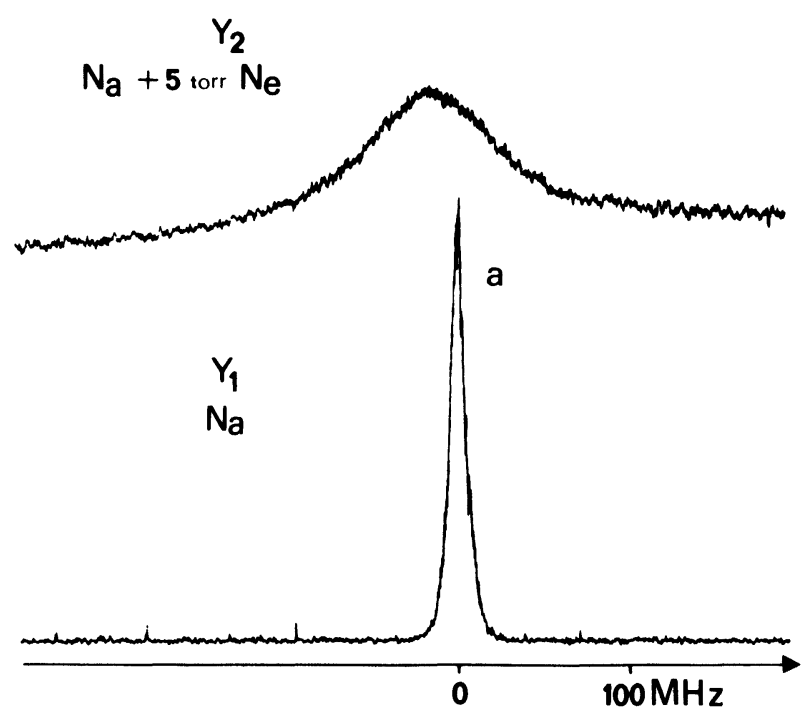

FIG. 3. - Enregistrement simultané de l'excitation à deux photons pour une cellule de $\mathrm{Na}$ sans gaz étranger (voie $\mathrm{Y}_{1}$ ) et pour une cellule contenant $\mathrm{Na}$ et 5 torr de $\mathrm{Ne}$ (voie $\mathrm{Y}_{2}$ ). La résonance (a) correspond à une transition du sous-niveau hyperfin $F=2$ du fondamental vers le niveau $J^{\prime}=\frac{5}{2}$ de l'état excité. On voit sur cet enregistrement qu'en présence de néon la résonance se déplace vers des fréquences plus petites, donc vers des longueurs d'onde plus grandes.

dilatée la résonance (a) simultanément enregistrée en absence de néon sur la voie $Y_{1}$ et en présence de 5 torr de néon sur la voie $Y_{2}$. La résonance sans largeur Doppler (mais élargie par collision), enregistrée sur la voie $Y_{2}$, se détache sur un fond en légère déclivité ; cette déclivité représente l'aile de la raie élargie par effet Doppler qui correspond à l'absorption de deux photons se propageant dans le même sens. (L'amplification moindre utilisée sur la voie $Y_{1}$ rend cette large raie Doppler invisible.)

A la précision de nos mesures, aucun écart significatif n'a été noté entre les élargissements ou les déplacements des diverses résonances (a), (b), (c) et (d) : pour une pression donnée, ces résonances ont même largeur et même déplacement. Il convient également de noter que l'on n'a décelé aucune asymétrie sur les transitions considérées et que pour une pression de néon de 1 torr ou 5 torr les raies de résonance sont des lorentziennes. Pour des pressions inférieures cela n'est plus vrai; en effet la forme de la résonance est le résultat de la convolution d'une lorentzienne et de la fonction d'appareil (jitter du LASER) dont les largeurs sont comparables. La fonction d'appareil est une courbe dont les ailes décroissent plus rapidement que celles d'une lorentzienne, mais dont le comportement au voisinage du centre (pour une abscisse pouvant valoir deux ou trois largeurs naturelles) est par contre très semblable à une lorentzienne.

Sur la figure 4 on a porté la largeur $L(p)$ de la résonance ainsi que le déplacement $D(p)$ du centre de la résonance en fonction de la pression $p$ de néon (nous avons fait des mesures pour $p=0,2$ torr, 1 torr et 5 torr). Les largeurs et les déplacements indiqués 


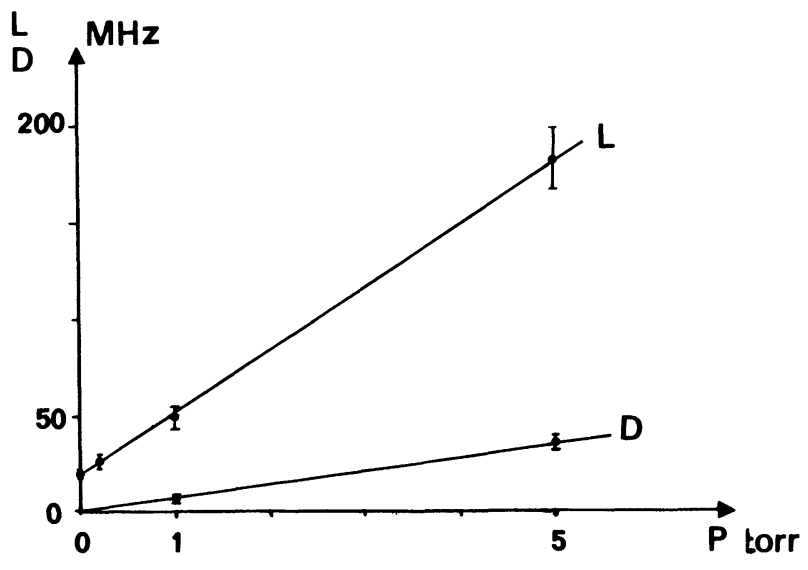

Fig. 4. - Valeur absolue du déplacement et élargissement en fonction de la pression de néon. Ces quantités sont relatives aux fréquences de Bohr de l'atome : elles sont deux fois plus grandes que les grandeurs mesurées sur les figures 2 et 3 .

sur cette figure 4 sont ceux des fréquences de Bohr de l'atome et non plus, comme dans le cas des figures 2 et 3, de la fréquence du LASER. Comme la transition est une absorption à deux photons, il faut multiplier par deux les largeurs et les déplacements mesurés sur les figures 2 et 3 , pour retrouver les quantités portées en ordonnée sur la figure 4 . On déduit de ces expériences les coefficients

$$
\begin{aligned}
& \alpha=\frac{L(p)-L(0)}{p}=32 \pm 5 \mathrm{MHz} / \text { torr } \\
& \beta=\frac{D(p)}{p}=-7 \pm 1 \mathrm{MHz} / \text { torr }
\end{aligned}
$$

(la pression de néon étant mesurée à $20^{\circ} \mathrm{C}$ ).

Ces résultats sont les tous premiers d'un ensemble d'expérience où nous projetons de faire une étude systématique de l'influence des collisions sodium-gaz rares sur les transitions à deux photons 3S-4D et 3S-5S dans l'atome de sodium. Ils démontrent les possibilités nouvelles offertes par ces transitions sans élargissement Doppler pour l'étude des collisions à de faibles pressions.

\section{Bibliographie}

[1] Biraben, F., Cagnac, B. et Grynberg, G., Phys. Rev. Lett. 32 (1974) 643.

[2] Levenson, M. D. and Bloembergen, N., Phys. Rev. Lett. 32 (1974) 645.

[3] Hansch, T. W., Harvey, K., Meisel, G. et Schawlow, A. L., Opt. Commun. 11 (1974) 50.

[4] Vasilenko, L. S., Chebotayev, V. P. et Shishaev, A. V., J.E.T.P. Lett. 11 (1970) 113.

[5] Cagnac, B., Grynberg, G. et Biraben, F., J. Physique 34 (1973) 845.

[6] Levenson, M. D. et Salour, M. M., Phys. Lett. 48A (1974) 331.
[7] Biraben, F., Cagnac, B. et Grynberg, G., Phys. Lett. 48A (1974) 469.

[8] Biraben, F., Cagnac, B. et Grynberg, G., Phys. Lett. 49A (1974) 71.

[9] On trouvera des références sur l'élargissement et le déplacement des raies de résonance dans l'article de revue suivant :

SChuller, F. et Behmenburg, W., Phys. Lett. 12C (1974) 273.

[10] Pritchard, D., Apt, J. et Ducas, T. W., Phys. Rev. Lett. 32 (1974) 641.

[11] Biraben, F., Cagnac, B. et Grynberg, G., C. R. Hebd. Séan. Acad. Sci. 279 (1974) B 51. 